\title{
Biologic Agents in Behçet's Disease: Our Experience and Review of the Literature
}

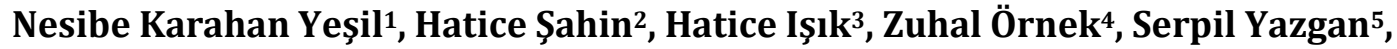 \\ Adem Tok ${ }^{6}$, Yunus Emre Yandı ${ }^{7}$, Metin Işık ${ }^{8}$, İsmail Doğan ${ }^{9}$ \\ ${ }^{1}$ Department of Rheumatology, Ankara Research and Education Hospital, Ankara, Turkey \\ ${ }^{2}$ Department of Internal Medicine, Faculty of Medicine, Bülent Ecevit University, Zonguldak, Turkey \\ ${ }^{3}$ Department of Obstetrics and Gynecology, Faculty of Medicine, Bülent Ecevit University, Zonguldak, Turkey \\ ${ }^{4}$ Department of Pediatric Disaease, Faculty of Medicine, Bülent Ecevit University, Zonguldak, Turkey \\ ${ }^{5}$ Department of Ophtalmology, Faculty of Medicine, Bülent Ecevit University, Zonguldak, Turkey \\ ${ }^{6}$ Department of Urology, Faculty of Medicine, Bülent Ecevit University, Zonguldak, Turkey \\ ${ }^{7}$ Division of Biochemistry, Department of Medical Services and Techniques, Health Sciences, Vocational School, \\ Bülent Ecevit University, Zonguldak, Turkey \\ ${ }^{8}$ Division of Rheumatology, Department of Internal Medicine, Faculty of Medicine, Bülent Ecevit University, \\ Zonguldak, Turkey \\ ${ }^{9}$ Division of Rheumatology, Department of Internal Medicine, Faculty of Medicine, Hitit University, Çorum, \\ Turkey \\ Email: "metin1721978@yahoo.com
}

Received 24 September 2015; accepted 24 October 2015; published 27 October 2015

Copyright (C) 2015 by authors and Scientific Research Publishing Inc.

This work is licensed under the Creative Commons Attribution International License (CC BY).

http://creativecommons.org/licenses/by/4.0/

\section{Abstract}

Behçet's disease (BD) is a large vessel vasculitis with a wide range of clinical manifestations. Some of these manifestation may be life threatening and rapid suppression of the inflammation with effective immunosuppressive agent is crucial. There are traditional drugs with different response rates and all have efficacy on different manifestations of the disease. The most frightening manifestations of the disease are ocular, neurologic, intestinal and vascular types of involvement. Besides benign and easily treated manifestations there are also refractory cases with complicated involvement. The novel biologic agents have been used for these resistant patients and favorable response rates have been reported. In this review, we have shared our experience with biologic agents in BD and also reviewed the literature for the efficacy and safety for these novel agents for refractory patients.

\footnotetext{
${ }^{*}$ Corresponding author.
}

How to cite this paper: Yeşil, N.K., Şahin, H., Işık, H., Örnek, Z., Yazgan, S., Tok, A., Yandı, Y.E., Işık, M. and Doğan, İ. (2015) Biologic Agents in Behçet's Disease: Our Experience and Review of the Literature. Open Journal of Rheumatology and Autoimmune Diseases, 5, 97-103. http://dx.doi.org/10.4236/ojra.2015.54016 


\section{Keywords}

\section{Behçet's Disease, Novel Biologic Agents, Refractory Patients}

\section{Introduction}

Behçet's disease (BD) is a systemic large-vessel vasculitis with various clinical manifestations like recurrent oral and genital aphthous ulcerations, uveitis, vascular, neurological, articular and gastrointestinal manifestations [1] [2]. Management of BD depends on the severity and the type of the clinical manifestation. Colchicine and some nonsteroidal anti-inflammatory agents are generally efficacious for muco-cutaneous and joint symptoms [3]. On the other hand, for patients with posterior uveitis, vasculitis, and patients with neurological and gastrointestinal involvements, aggressive immunosuppressive agents are necessary [4]. Uveitis is the most frequent complication of BD and besides interferon alfa, infliximab, adalimumab, anakinra, canakinumab, tocilizumab and ruti-ximab are the novel agents for resistant diseases with favorable efficacy and tolerability [5]. Long-term remission, protection of visual acuity and lowering systemic corticosteroid necessity are the main treatment targets in ocular BD. On the other hand, BD may present with neurologic symptoms as parenchymal or nonparenchymal neuro-Behçet disease (NBD). Parenchymal NBD is more frequent and defined as an inflammatory perivasculitis, in the brainstem as subacute meningoencephalitis or may present as unilateral lesion in the upper brainstem extending into the thalamus and basal ganglia. Venous sinus thrombosis is the most common presentation of the second form of NBD and headache and papilledema are the most frequent signs [6]. High dose corticosteroid therapies for attacks and azathioprine, cyclophosphamide, interferon- $\alpha$ and anti-TNF agents for longterm preventive treatment are the general approach to NBD but trials are limited to build up a guideline [7]. Similarly, for patients with gastrointestinal manifestations 5-aminosalicylic acid, corticosteroids, immune-modulators, and anti-tumor necrosis factor alpha monoclonal antibody therapy are prescribed [8].

Herein, we have reported our experience with biologic agents in BD and also tried to review the literature. All the accessible trials or manuscripts about biologic therapy in BD were included.

\section{Patient Characteristics in Our Study Population}

There were totally 128 patients with $\mathrm{BD}$ in our medical database but only 108 were suitable to be classified as BD according to the International Study Group criteria. Of all, 41 (38\%) male and 67 (62\%) female patients with a median age of $41.5(16$ - 68) were analyzed. The median disease duration was $76(0$ - 327) months. The frequencies of disease manifestation were as follows; oral apthous ulcers; $100 \%$, genital ulcers; $82.4 \%$, erythema nodosum; 43.5\%, ocular involvement; 25.9\%, parenchymal NBD; 4.6\%, venous sinus thrombosis; 0.9\%, gastrointestinal involvement; 1.9\%, arterial inflammation (aortitis and arterial aneurysms); 5.6\%, arthritis; $18.5 \%$, deep vein thrombosis; $15.7 \%$ and other type of skin manifestations; $22.2 \%$. Pathergy skin test was done for 28 patients and $60.7 \%$ were positive for this test. Human leucocyte antigen B 51 (HLA B51) was studied in 16 patients and $68.7 \%$ were positive. Colchicine was prescribed to all patients and the second most frequently used agent was azathioprine (29.6\%). Six (5.5\%) patients have used intravenous pulse steroids and cyclophosphamide therapy, 5 for parenchymal NBD and 3 for aortitis (2 patients had concomitant aortitis and NBD).

Seventeen (15.7\%) patients have used interferon alpha 2a, 12 for ocular involvement, 3 for arterial aneurysms (vasculitis) 1 for parenchymal NBD and 1 for gastrointestinal disease. Eleven patient have taken anti-TNFagents; 8 infliximab, 1 golimumab and 1 adalimumab. One patient has used multiple anti-TNF agents; etanercept, adalimumab and infliximab in order. Eight patients with ocular disease, 1 with parenchymal NBD, 1 with gastrointestinal disease and 1 with resistant arthritis were on Anti-TNF therapy.

In our study population, anakinra, tocilizumab, canakinumab and rutiximab were not used.

\section{Biologic Agents for Behçet's Disease}

\subsection{Ocular Disease}

Ocular involvement in BD is a diagnostic criteria according to the international study group and also is one of the most frequent manifestation. Non granulomatous type of anterior uveitis sometimes with hypopion or post- 
erior involvement including vitritis, retinal infiltrates, sheathing of retinal veins, occlusive vasculitis, and macular edema are the main presentations of ocular disease. Male patients, especially younger ones, have a worse prognosis and visual loss is more frequent among this group. Current treatment goals for ocular disease are rapid suppression of intraocular inflammation, preservation of vision, prevention of recurrences, and achievement of remission. For patient with posterior uveitis and retinal vasculitis, interferon alpha and TNF alpha blockers have successfully improved the visual prognosis and after the usage of these two agents for refractory cases, blindness is no more a common problem.

With the beginning of the millennium, efficacy of interferon alpha for refractory ocular disease has been reported and in 2008 EULAR has also recommended IFN alpha for patients with refractory disease in the first step. The major adverse effects of IFN alpha are cytopenia and depression.

Kotter I. et al., have reported that $94 \%$ of the patients with active uveitis have benefit from IFN alpha in the first 4 weeks and more than half of the patients had long-term remission after 15 months of therapy. Revascularization of the retinal vein and regression of neovascularization were also observed [9]. Deuter C.M. et al., have reported 98.1\% response rates INF alpha and 50\% drug free remission after 4 years. The recovery in visual acuity was long lived and women had better prognosis. Furthermore, IFN alpha 2a was reported to be more effective than IFN alpha 2b [10]. In a systematic review by Kotter I. in 2004, 338 patient treated with interferon alpha were reviewed and $94 \%$ of the patients with uveitis had partial or complete response [11].

Another important biologic agent for the ocular involvement is infliximab. In a multicenter analysis from Japan, 164 patients with refractory uveitis on infliximab therapy were evaluated and a prominent decrease in number of attacks was provided, as well as a 55\% increase in best visual acuity [12].

Keino H. et al., reported 9 patients with Behçet's uveitis and stated that infliximab reduced ocular attacks and mean background retinal/disc vascular leakage. Similarly, Kaburaki T. et al., have reported decrease in number and severity of attacks [13] [14]. Okada A.A. and collegues have evaluated the safety of infliximab for uveitis in $\mathrm{BD}$ and have reported that infliximab was very effective and also safe for these patients [15].

Perra D. et al., have used adalimumab for refractory BD and all the 8 patients with Behçets uveitis and retinal vasculitis responded adalimumab rapidly [16].

Arida A. et al., reviewed the literature for anti-TNF in BD and found that etanercept, adalimumab and infliximab were all effective and safe options for refractory patients with BD [17]. In 2014, the expert panel recommendations were published and both infliximab and adalimumab were recommended as first line therapy for patients with Behçets uveitis and switch between two agents were accepted to be safe and effective to keep remission [18].

Blockage of IL-1 and 6 may also work in refractory Behçets uveitis because these two cytokines take place in the pathophysiology of the disease but there are no adequate data. Tocilizumab is an IL-6 blocker and have been used for takayasu and giant cell arteritis but not for Behçet uveitis. On the other hand, there are some data showing that tocilizumab has favorable response in NBD [19].

In conclusion, for refractory ocular disease IFN alpha 2a, infliximab and perhaps adalimumab may be effective treatment options but further trials are necessary with other novel biologic agents.

\subsection{Neuro-Behçet}

Neurologic manifestations of BD are not as common as ocular manifestations but are life threatening and may cause severe disability. Therefore, NBD should be treated rapidly and effectively as well. For years high dose corticosteroids and immunosuppressive agents were prescribed but novel biologic agents are also effective [7].

Borhani Haghighi A. et al., reported 4 patients with NBD and 2 patients on $3 \mathrm{mg} / \mathrm{kg}$ dose without any adjacent agent had unfavorable results but the other two who took $5 \mathrm{mg} / \mathrm{kg}$ dose and monthly cyclophosphamide besides achieved good response [20].

Kanemura H. et al., reported a patient with good response to infliximab with long-term follow up [21]. Hirohata S. et al., studied the cytokines responsible for the development of NBD and reported that especially IL 6 and 8 levels were increased in the cerebrospinal fluid, which may mean that blockade of these two cytokines may be other future therapies for NBD [22]. Shapiro L.S. and collegue have reported a refractory NBD patient with an excellent response to IL-6 blocker tocilizumab [23].

Although not adequate, experience with INF alpha is also present for NBD. For dural sinus thrombosis with headache and increase in intracranial pressure a brief course of steroid therapy is recommended [24]. In our 
study group, all the patients with parenchymal NBD had taken pulse corticosteroid and intravenous cyclophosphamide therapy, and for remission induction and for maintenance of remission other immune-suppressive agents were preferred. Therefore, further trials are necessary and therapy decision must be tailored to the patient's characteristic features.

\subsection{Entero-Behçet}

Gastrointestinal involvement in BD may be complicated by serious comorbidities, such as gastrointestinal bleeding and perforation, with poor prognosis. For decades high-dose corticosteroids (CSs) and immuno-suppressants were widely used for treatments, but refractory or steroid depended entero-BD was difficult to be managed, and effective treatment regimens are necessary. Biologic agents may be an option for these refractory patients.

There are some reports declaring response to infiliximab plus thalidomide or infliximab plus methotrexate for patients with refractory entero-BD and also a small study is also present with infliximab [25]-[31]. On the other hand, case reports with etanercept, interferon and a trial with adalimumab are also present but tocilizumab was not sufficiently investigated for entero-BD [32]-[37]. In our study population, there were only 2 patients with entero-behçets disease and one was treated with infliximab, colchicine and steroid combination and the other one with interferon and azathioprine combination. Both had favorable responses.

\subsection{Major Vessel Disease}

Although BD is known as a large vessel vasculitis, all vessels of any size may be affected. Thrombosis and arterials aneurysms are common and anti-coagulation with immune-suppression for venous thrombosis is recommended. For arterial disease high dose corticosteroids with other immune-suppressive agents are necessary [24].

Schäfer VS has reviewed the literature for biologic agents in large vessel vasculitis and have recommended usage of anti-TNF agents and tocilizumab for refractory patients [38].

Adler and colleagues reported 7 patients with vascular BD responding to infliximab, three with aortic involvement, one with recurrent venous thrombosis of the pelvic veins, one with recurrent venous and arterial thromboses of the thigh, and two with retinal vasculitis. Inflammation has been rapidly suppressed and infliximab was found to be effective for both inducing and maintaining remission in vascular BD and the authors have recommended infliximab for refractory patients with vascular manifestations [39]. Lee et al, in 2010 have tested adalimumab for bilateral pulmonary arterial aneurysm and reported acceptable response [40].

In our study population, there were 6 patients with arterial involvenment and 17 patients with venous disease. Three of them were treated with intravenous pulse steroid plus cyclophosphamide combination and 3 with high dose corticosteroids plus azathioprine combinations. Patients with venous disease used colchicine, low dose steroids and azathioprine $(13 / 17 ; 76.4 \%)$.

\subsection{Joint Involvement}

Arthritis in BD is usually mild and non-deforming type of arthritis mostly involving the knee. Colchicine is mostly adequate for arthritis in BD and rarely sulfasalazine and methotrexate with low dose steroid have been used. Biologic agents are very rarely necessary. Donghi D has reported a very refractory patient with arthritis, NBD, uveitis and entero-behçet's disease treated with infliximab [41]. On the other hand, ankylosing spondylitis may accompany BD and for these patients anti-TNF agents are prescribed but for purely behçets arthritis colchicine is mostly enough. In our study population there were 20 patients with arthritis and 8 of them had accompanying ankylosing spondylitis and other 12 patients were on colchicine therapy ( \pm low dose corticosteroids).

\section{Conclusions}

Behçet's disease is common in the old silk-road and as a vasculitis a wide range of clinical manifestations may complicate the disease. Some patients with ocular, intestinal, vascular and neurologic involvement may be refractory to traditional immunosuppressive agents or may become high dose steroid depended; for these refractory patients biologic agent may provide benefit. The most frequently prescribed and most widely studied agent is interferon alpha 2a but infliximab and to a lesser extent other anti-TNF agents, rutiximab, tocilizumab, canakinumab, rutiximab and anakinra may all provide acceptable response rates for selected patients; and of course, 
further randomized placebo controlled trials are necessary for further recommendations and to build up guidelines. In the near future, alemtuzumab and hematopoietic stem cell transplantation may also be studied in complicated and refractory behçets disease.

To our opinion, biologic agents should be preserved for patients resistant to all traditional immunosuppressive agents or for high dose steroid dependent patients. The clinical feature and personal characteristics of the patient, experience of the clinician with the biologic agent, safety and efficacy data are all important factors. Finally, we recommend the clinicians to be brave and self-confident in using biologic agents but careful as well.

\section{References}

[1] Arayssi, T. and Hamdan, A. (2004) New Insights into the Pathogenesis and Therapy of Behcet's Disease. Current Opinion in Pharmacology, 4, 183-188. http://dx.doi.org/10.1016/j.coph.2003.10.009

[2] Sakane, T., Takeno, M., Suzuki, N. and Inaba, G. (1990) Behcet’s Disease. New England Journal of Medicine, 341, 1284-1291. http://dx.doi.org/10.1056/NEJM199910213411707

[3] Yurdakul, S., Mat, C., Tuzun, Y., Ozyazgan, Y., Hamuryudan, V., Uysal, O., et al. (2001) A Doubleblind Trial of Colchicine in Behcet’s Syndrome. Arthritis \& Rheumatology, 44, 2686-2692. http://dx.doi.org/10.1002/1529-0131(200111)44:11<2686::AID-ART448>3.0.CO;2-H

[4] Comarmond, C., Wechsler, B., Bodaghi, B., Cacoub, P. and Saadoun, D. (2014) Biotherapies in Behçet’s Disease. Autoimmunity Reviews, 13, 762-769. http://dx.doi.org/10.1016/j.autrev.2014.01.056

[5] Mesquida, M., Molins, B., Llorenç, V., Hernández, M.V., Espinosa, G., Dick, A.D. and Adán, A. (2014) Current and Future Treatments for Behçet’s Uveitis: Road to Remission. International Ophthalmology, 34, 365-381. http://dx.doi.org/10.1007/s10792-013-9788-5

[6] Fountain, E.M. and Dhurandhar, A. (2014) Neuro-Behçet’s Disease: An Unusual Cause of Headache. Journal of General Internal Medicine, 29, 956-960. http://dx.doi.org/10.1007/s11606-014-2781-5

[7] Saip, S., Akman-Demir, G. and Siva, A. (2014) Neuro-Behçet Syndrome. Handbook of Clinical Neurology, 121, 17031723. http://dx.doi.org/10.1016/B978-0-7020-4088-7.00110-3

[8] Chin, A.B. and Kumar, A.S. (2015) Behcet Colitis. Clinics in Colon and Rectal Surgery, 28, 99-102. http://dx.doi.org/10.1055/s-0035-1547336

[9] Kotter, I., Zierhut, M., Eckstein, A., Vonthein, R., Ness, T., Gunaydin, I., et al. (2003) Human Recombinant Interferon Alfa-2a for the Treatment of Behcet's Disease with Sight Threatening Posterior or Panuveitis. British Journal of Ophthalmology, 87, 423-431. http://dx.doi.org/10.1136/bjo.87.4.423

[10] Deuter, C.M., Zierhut, M., Möhle, A., Vonthein, R., Stöbiger, N. and Kötter, I. (2010) Long-Term Remission after Cessation of Interferon- $\alpha$ Treatment in Patients with Severe Uveitis Due to Behçet's Disease. Arthritis \& Rheumatology, 62, 2796-2805. http://dx.doi.org/10.1002/art.27581

[11] Kotter, I., Gunaydin, I., Zierhut, M. and Stubiger, N. (2004) The Use of Interferon Alpha in Behcet Disease: Review of the Literature. Seminars in Arthritis and Rheumatism, 33, 320-335. http://dx.doi.org/10.1016/j.semarthrit.2003.09.010

[12] Takeuchi, M., Kezuka, T., Sugita, S., Keino, H., Namba, K., Kaburaki, T., Maruyama, K., Nakai, K., Hijioka, K., Shibuya, E., Komae, K., Hori, J., Ohguro, N., Sonoda, K.H., Mizuki, N., Okada, A.A., Ishibashi, T., Goto, H. and Mochizuki, M. (2014) Evaluation of the Long-Term Efficacy and Safety of Infliximab Treatment for Uveitis in Behçet's Disease: A Multicenter Study. Ophthalmology, 121, 1877-1884. http://dx.doi.org/10.1016/j.ophtha.2014.04.042

[13] Keino, H., Okada, A.A., Watanabe, T. and Taki, W. (2014) Long-Term Efficacy of Infliximab on Background Vascular Leakage in Patients with Behçet’s Disease. Eye, 28, 1100-1106. http://dx.doi.org/10.1038/eye.2014.138

[14] Kaburaki, T., Namba, K., Sonoda, K.H., Kezuka, T., Keino, H., Fukuhara, T., Kamoi, K., Nakai, K., Mizuki, N. and Ohguro, N., Ocular Behçet Disease Research Group of Japan (2014) Behçet’s Disease Ocular Attack Score 24: Evaluation of Ocular Disease Activity before and after Initiation of Infliximab. Japanese Journal of Ophthalmology, 58, 120130. http://dx.doi.org/10.1007/s10384-013-0294-0

[15] Okada, A.A., Goto, H., Ohno, S. and Mochizuki, M., Ocular Behçet’s Disease Research Group of Japan (2012) Multicenter Study of Infliximab for Refractory Uveoretinitis in Behçet Disease. Archives of Ophthalmology, 130, 592-598. http://dx.doi.org/10.1001/archophthalmol.2011.2698

[16] Perra, D., Alba, M.A., Callejas, J.L., Mesquida, M., Ríos-Fernández, R., Adán, A., Ortego, N., Cervera, R. and Espinosa, G. (2012) Adalimumab for the Treatment of Behcet’s Disease: Experience in 19 Patients. Rheumatology, 51, 1825-1831. http://dx.doi.org/10.1093/rheumatology/kes130

[17] Arida, A., Fragiadaki, K., Giavri, E. and Sfikakis, P.P. (2011) Anti-TNF Agents for Behçet’s Disease: Analysis of Published Data on 369 Patients. Seminars in Arthritis and Rheumatism, 41, 61-70. http://dx.doi.org/10.1016/j.semarthrit.2010.09.002 
[18] Levy-Clarke, G., Jabs, D.A., Read, R.W., Rosenbaum, J.T., Vitale, A. and Van Gelder, R.N. (2014) Expert Panel Recommendations for the Use of Anti-Tumor Necrosis Factor Biologic Agents in Patients with Ocular Inflammatory Disorders. Ophthalmology, 121, 785-796.e3. http://dx.doi.org/10.1016/j.ophtha.2013.09.048

[19] Urbaniak, P., Hasler, P. and Kretzschmar, S. (2012) Refractory Neuro-Behçet Treated by Tocilizumab: A Case Report. Clinical and Experimental Rheumatology, 30, S73-S75.

[20] Haghighi, A.B., Safari, A., Nazarinia, M.A., Habibagahi, Z. and Shenavandeh, S. (2011) Infliximab for Patients with Neuro-Behcet’s Disease: Case Series and Literature Review. Clinical Rheumatology, 30, 1007-1012. http://dx.doi.org/10.1007/s10067-011-1726-1

[21] Kanemaru, H., Makino, T., Jinnin, M., Yonemitsu, A., Makino, K. and Ihn, H. (2013) Case of Neuro-Behçet’s Disease Successfully Maintained a Remission Using Infliximab for 2 Years. The Journal of Dermatology, 40, 632-634. http://dx.doi.org/10.1111/1346-8138.12203

[22] Hirohata, S. and Kikuchi, H. (2012) Changes in Biomarkers Focused on Differences in Disease Course or Treatment in Patients with Neuro-Behçet's Disease. Internal Medicine, 51, 3359-3365. http://dx.doi.org/10.2169/internalmedicine.51.8583

[23] Shapiro, L.S., Farrell, J. and Haghighi, A.B. (2012) Tocilizumab Treatment for Neuro-Behcet's Disease, the First Report. Clinical Neurology and Neurosurgery, 114, 297-298. http://dx.doi.org/10.1016/j.clineuro.2011.10.024

[24] Hatemi, G., Silman, A., Bang, D., Bodaghi, B., Chamberlain, A.M., Gul, A., Houman, M.H., Kötter, I., Olivieri, I., Salvarani, C., Sfikakis, P.P., Siva, A., Stanford, M.R., Stübiger, N., Yurdakul, S. and Yazici, H., EULAR Expert Committee (2008) EULAR Recommendations for the Management of Behçet Disease. Annals of the Rheumatic Diseases, 67, 1656-1662. http://dx.doi.org/10.1136/ard.2007.080432

[25] Sfikakis, P.P., Markomichelakis, N., Alpsoy, E., Assaad-Khalil, S., Bodaghi, B., Gul, A., Ohno, S., Pipitone, N., Schirmer, M., Stanford, M., Wechsler, B., Zouboulis, C., Kaklamanis, P. and Yazici, H. (2007) Anti-TNF Therapy in the Management of Behcet's Disease—-Review and Basis for Recommendations. Rheumatology, 46, 736-741. http://dx.doi.org/10.1093/rheumatology/kem034

[26] Travis, S.P., Czajkowski, M., McGovern, D.P., Watson, R.G. and Bell, A.L. (2001) Treatment of Intestinal Behçet’s Syndrome with Chimeric Tumour Necrosis Factor Alpha Antibody. Gut, 49, 725-728. http://dx.doi.org/10.1136/gut.49.5.725

[27] Hassard, P.V., Binder, S.W., Nelson, V. and Vasiliauskas, E.A. (2001) Anti-Tumor Necrosis Factor Monoclonal Antibody Therapy for Gastrointestinal Behçet’s Disease: A Case Report. Gastroenterology, 120, 995-999. http://dx.doi.org/10.1053/gast.2001.22556

[28] Naganuma, M., Sakuraba, A., Hisamatsu, T., Ochiai, H., Hasegawa, H., Ogata, H., Iwao, Y. and Hibi, T. (2008) Efficacy of Infliximab for Induction and Maintenance of Remission in Intestinal Behçet’s Disease. Inflammatory Bowel Diseases, 14, 1259-1264. http://dx.doi.org/10.1002/ibd.20457

[29] Iwata, S., Saito, K., Yamaoka, K., Tsujimura, S., Nawata, M., Suzuki, K. and Tanaka, Y. (2009) Effects of Anti-TNFAlpha Antibody Infliximab in Refractory Entero-Behcet’s Disease. Rheumatology, 48, 1012-1013. http://dx.doi.org/10.1093/rheumatology/kep126

[30] Kinoshita, H., Kunisaki, R., Yamamoto, H., Matsuda, R., Sasaki, T., Kimura, H., Tanaka, K., Naganuma, M. and Maeda, S. (2013) Efficacy of Infliximab in Patients with Intestinal Behçet’s Disease Refractory to Conventional Medication. Internal Medicine, 52, 1855-1862. http://dx.doi.org/10.2169/internalmedicine.52.0589

[31] Lee, J.H., Cheon, J.H., Jeon, S.W., Ye, B.D., Yang, S.K., Kim, Y.H., Lee, K.M., Im, J.P., Kim, J.S., Lee, C.K., Kim, H.J., Kim, E.Y., Kim, K.O., Jang, B.I. and Kim, W.H. (2013) Efficacy of Infliximab in Intestinal Behçet’s Disease: A Korean Multicenter Retrospective Study. Inflammatory Bowel Diseases, 19, 1833-1838.

[32] De Cassan, C., De Vroey, B., Dussault, C., Hachulla, E., Buche, S. and Colombel, J.F. (2011) Successful Treatment with Adalimumab in a Familial Case of Gastrointestinal Behcet's Disease. Journal of Crohn's and Colitis, 5, 364-368. http://dx.doi.org/10.1016/j.crohns.2011.03.006

[33] Ariyachaipanich, A., Berkelhammer, C. and Nicola, H. (2009) Intestinal Behçet’s Disease: Maintenance of Remission with Adalimumab Monotherapy. Inflammatory Bowel Diseases, 15, 1769-1771. http://dx.doi.org/10.1002/ibd.20869

[34] van Laar, J.A., Missotten, T., van Daele, P.L., Jamnitski, A., Baarsma, G.S. and van Hagen, P.M. (2007) Adalimumab: A New Modality for Behçet's Disease? Annals of the Rheumatic Diseases, 66, 565-566. http://dx.doi.org/10.1136/ard.2006.064279

[35] Shimizu, Y., Takeda, T., Matsumoto, R., Yoshida, K., Nakajima, J., Atarashi, T., Yanagisawa, H., Kikuchi, K. and Kikuchi, H. (2012) Clinical Efficacy of Adalimumab for a Postoperative Marginal Ulcer in Gastrointestinal Behçet Disease. Nihon Shokakibyo Gakkai Zasshi, 109, 774-780.

[36] Sylwestrzak, G., Liu, J., Stephenson, J.J., Ruggieri, A.P. and De Vries, A. (2014) Considering Patient Preferences When Selecting Anti-Tumor Necrosis Factor Therapeutic Options. Am Health Drug Benefits, 7, 71-81. 
[37] Tanida, S., Inoue, N., Kobayashi, K., Naganuma, M., Hirai, F., Iizuka, B., Watanabe, K., Mitsuyama, K., Inoue, T., Ishigatsubo, Y., Suzuki, Y., Nagahori, M., Motoya, S., Nakamura, S., Arora, V., Robinson, A.M., Thakkar, R.B. and Hibi, T. (2015) Adalimumab for the Treatment of Japanese Patients with Intestinal Behçet’s Disease. Clinical Gastroenterology and Hepatology, 13, 940-948.e3. http://dx.doi.org/10.1016/j.cgh.2014.08.042

[38] Schäfer, V.S. and Zwerina, J. (2012) Biologic Treatment of Large-Vessel Vasculitides. Current Opinion in Rheumatology, 24, 31-37. http://dx.doi.org/10.1097/BOR.0b013e32834dc392

[39] Adler, S., Baumgartner, I. and Villiger, P.M. (2012) Behçet’s Disease: Successful Treatment with Infliximab in 7 Patients with Severe Vascular Manifestations. A Retrospective Analysis. Arthritis Care \& Research, 64, 607-611. http://dx.doi.org/10.1002/acr.21557

[40] Lee, S.W., Lee, S.Y., Kim, K.N., Jung, J.K. and Chung, W.T. (2010) Adalimumab Treatment for Life Threatening Pulmonary Artery Aneurysm in Behçet Disease: A Case Report. Clinical Rheumatology, 29, 91-93. http://dx.doi.org/10.1007/s10067-009-1272-2

[41] Donghi, D. and Mainetti, C. (2010) Infliximab for the Treatment of Refractory Adamantiades-Behçet Disease with Articular, Intestinal, Cerebral and Ocular Involvement. Dermatology, 220, 282-286.

http://dx.doi.org/10.1159/000277928 